Historia y comunicación social

ISSN: $1137-0734$

\title{
Aprendiendo a amar: la evolución en el desarrollo dramático de los dating shows en España $(1992-2010)^{1}$
}

\author{
José Cabeza San Deogracias²; Raúl Casado Linares ${ }^{3}$
}

Recibido el: 18 de diciembre de 2019 / Aceptado: 2 de diciembre de 2020

Resumen. Este artículo muestra la evolución en el dating show en España entre 1992 y 2010. Se estudian los cambios en la estructura de los programas más vistos de este subgénero del reality, que pasó de preguntar curiosidades sobre las relaciones amorosas a comprobar y desafiar su grado de complicidad En estos años, los participantes de los dating shows estuvieron condicionados por las decisiones que tomaban las productoras para hacer mejores productos de entretenimiento, pero también tuvieron libertad para mostrar su intimidad y su espontaneidad dentro de los límites definidos por el reality. Paradójicamente, hasta que las personas no aprendieron a actuar dentro de un formato y asumieron su condición de personajes, estos programas no lograron más profundidad y realismo.

Palabras clave: Dating show; Reality show; Historia de la televisión; Audiencias; Hipertelevisión.

[en] Love is on the air: the evolution of dramatic development in Spanish dating shows (1992-2010)

\begin{abstract}
This paper shows the evolution of the dating shows in Spain between 1992 and 2010. We study their structural changes based on the most-viewed programs of this sub-genre, which developed from initially exploring innocent relationship anecdotes towards eventually testing and challenging the relationship itself. In these years, the dating show participants were influenced by the broadcasters' choices aiming for entertainment, but they were also free to show intimacy and spontaneity within the reality-show boundaries. Paradoxically, it was only when participants learned to act in line with the format and assumed their role as characters that these programs achieved more depth and realism.
\end{abstract}

Keywords: Dating show; Reality show; History of television; Audiences; Hypertelevision.

Sumario: 1. Introducción y estado de la cuestión. 2. Metodología. 3. Los dating shows: audiencias y primeros éxitos. 4. Gran Hermano y su influencia en el dating show. 5. La autenticidad y el reality. 6. Conclusiones. 7. Referencias bibliográficas.

Cómo citar: Cabeza San Deogracias, J.; Casado Linares, R. (2021) Aprendiendo a amar: la evolución en el desarrollo dramático de los dating shows en España (1992-2010), Historia y comunicación social 26(1), 35-43.

\section{Introducción y estado de la cuestión}

Normalmente, un género está definido por su solidez, por unas características o límites que le dan forma, que lo hacen inconfundible respecto a otros géneros. La telerrealidad (reality y talk show), en cambio, se constituye como un género líquido (Imbert, 2008a), definido más por su capacidad de cambio y de invasión de formatos, temas y espacios que por su carácter único. A partir de la primera década del siglo XXI, la telerrealidad encuentra la conexión con la audiencia a partir de unos elementos sencillos pero de extraña combinación: las estrategias de la información y la ficción (docudrama) y la forma narrativa de los concursos. La realidad empieza a organizarse como la ficción, pero sin perder lo que la hace reconocible: la autenticidad, es decir, la

Esta investigación se ha realizado dentro del proyecto de investigación Historia de la Programación y de los Programas de Televisión en España (cadenas de ámbito estatal): de la desregulación al apagón analógico, 1990-2010. Referencia: CSO2015-66260-C4-1-P, financiado por el Ministerio de Economía y Competitividad (España). Enero 2015 - Julio 2020.

2 Universidad Rey Juan Carlos

E-mail: jose.cabeza@urjc.es

ORCID: https://orcid.org/0000-0003-1047-2733

3 Arnhem University of Applied Sciences, Netherlands

E-mail: r.casadolinares@han.nl

ORCID: https://orcid.org/0000-0002-6121-8279

Hist. comun. soc. 26(1) 2021: 35-43 
representación veraz del ser humano. El surgimiento y el éxito de Gran Hermano (GH) (Telecinco, ZeppelinEndemol, 2000-) asentó el género al mismo tiempo que lo atomizaba: cualquier programa podía evolucionar y asumir nuevos límites en la recreación de la realidad para competir por ser originales ante la audiencia. Los formatos se posicionaban en la parrilla exhibiendo una parte de la realidad (tema) y una forma entretenida de exponerlo (estructura). Un espacio trataba de buscar pareja (dating show); otro de demostrar capacidades artísticas (talent show) o de ser capaz de enfrentarse a situaciones límites mejor que otros (docugame de convivencia y de supervivencia). Algunos se ocupaban de lograr que la gente fuera más feliz (coaching show); otros de hacer pública la vida o la personalidad de los famosos en diferentes contextos (celebrity show).

León (2009: 15) establece una clasificación de realities con gran prudencia -"sin querer ser exhaustivo"-y recoge las siguientes categorías: Accidentes y crímenes, Ayuda social, Intervención en la vida de las personas (coaching), De convivencia y relaciones personales y, por último, De formación artística (talent show). Sin embargo, su trasposición a la realidad presenta problemas. Operación Triunfo (OT) (TVE y Telecinco, Gestmusic-Endemol, 2001-) está etiquetado como talent show, pero cuando los cantantes hablan de cómo se sienten por estar nominados o se bloquean por cantar una canción que no se les adapta bien y se lo cuentan al coach o al presentador... surge el coaching. El mayor problema de cualquier intento de clasificación en el ámbito de los realities reside en la "hiperhibridación genérica" (Gordillo, 2009: 187): aunque un formato tenga una combinación original de elementos, irremediablemente también presenta múltiples conexiones con otro tipo de géneros o formatos.

El dating show, como subgénero dentro de los formatos de realities, adolece de los mismos problemas de definición que cualquier otro programa de la hipertelevisión. Hidalgo-Mari (2018: 625) cita tres grandes bloques "clarificativos" en el subgénero: dating talk show (un candidato a encontrar pareja cuenta qué quiere y cómo es a través de una entrevista guiada), dating reality show (convivencia entre las posibles parejas), dating game show (hay competición y más análisis psicológico de los candidatos para poder elegir a una pareja). Dentro de los dating game shows habría también otra subdivisión: speed dating game shows (citas entre personas desconocidas) y dating experts game shows (citas en las que un consejero puede influir en la decisión de las personas). Bastantes programas reúnen elementos de los tres bloques y hacen que su asignación a una etiqueta u otra no revele demasiado sobre la identidad de cada formato. Estas invasiones de un bloque a otro se producen de forma natural, porque todos los formatos trabajan con la misma realidad, más exactamente con el espectáculo de lo real, es decir, la necesidad de exponer en un formato audiovisual destinado al entretenimiento los inicios de una relación amorosa, conservando su carga emocional, pero organizándola en torno a momentos que pueden producir cambios en la vida real de las personas (presentaciones, citas, consejos). Incluso las críticas que ven los dating shows como market meat también asumen que la audiencia está dirigida por un componente documental de ver qué pasa realmente en una relación (Gray, 2009).

\section{Metodología}

Este artículo hace una revisión de los dating shows más vistos en el periodo 1990-2010 según los datos de Kantar. Se filtraron los más de 51.000 registros de Kantar para seleccionar exclusivamente los programas clasificados en la categoría de "reality show". Posteriormente, se tuvo que ampliar la búsqueda a aquellos que estaban también en categorías como "concurso" o "entretenimiento". Por ejemplo, Su media naranja (Telecinco, Boomerang TV,1991-1995) era un programa catalogado como "concurso". Por último, se elaboró una tabla de dating shows y se extrajeron los porcentajes de audiencia estimados para este tipo de programas, así como sus principales características (sexo, edad y nivel socioeconómico).

El estudio llevado a cabo no es meramente descriptivo para establecer una clasificación o simplemente una cronología, sino que profundiza en el desarrollo dramático de los dating shows, es decir, en los elementos estructurales que cambian y que hacen evolucionar el subgénero, así como en el influjo de GH en la nueva generación de programas de estas características. Hay autores que han apuntado que los formatos creados a partir de GH significan una traición al "noble impulso" del documental (Hill; Palmer, 2002: 252), y desde el primer momento surgieron dudas ante la capacidad representativa de lo que veían los espectadores, porque ¿cuánto de real es la realidad televisiva? (Cummings, 2002). Pero la ficción se hace presente -y necesaria- en cualquier tipo de reality a través de la edición y de la estructura dramática, aunque se oculte para potenciar el efecto realidad y transmitir la sensación de que el espectador no ve nada construido (Kilborn, 1994: 423). De ahí que no se pueda desligar la forma en la que se cuentan los realities de la evolución de las series de ficción de los 80 , que lograron que hubiera un desarrollo de personajes sometidos a multitud de incidentes en las tramas que les hacían cambiar y crecer emocionalmente (Ellis, 2009; Corner, 2009).

Por todo ello, estudiamos la evolución de los dating shows según dos dimensiones. Por un lado, la ficción y las reglas o límites que impone, creando "un modelo narrativo abierto, no del todo guionizador" (Imbert, 2008b: 75). Y, por otro lado, la búsqueda de la autenticidad para crear una comunicación emocional entre la persona y la audiencia que espera encontrar verdad en los formatos de reality. 


\section{Los dating shows: audiencias y primeros éxitos}

En términos de audiencia, los dating shows obtenían aproximadamente un $25 \%$ de cuota de pantalla en la década de los 90 , pero este porcentaje bajó significativamente al $15 \%$ en la década siguiente. El descenso no se debió tanto a una pérdida de interés del público como a la proliferación de programas de tipo reality que surgieron como competencia por efecto de la hipertelevisión.

Demográficamente, este subgénero de los reality atrajo mayoritariamente a un público femenino $(63 \%$ de la audiencia fueron mujeres, $37 \%$ hombres), aunque estos porcentajes varían según el programa: en Mujeres $y$ hombres y viceversa (MyHyV) (Bulldog Producciones, 2008-), el porcentaje femenino sube hasta un 66\%, mientras que en Eligeme (Cuatro, Fremantle Media, 2009) y en Granjero busca esposa (Cuatro, Grundy Producciones, 2008-2011; 2016-), las ratios estuvieron equilibradas (51\% hombres, $49 \%$ de mujeres). Esta diferencia por sexo (Tabla 1) no sólo se produjo en términos absolutos (es decir, había más mujeres que hombres viendo el dating show), sino también relativos: el promedio de cuota de pantalla para este tipo de programas fue un $24 \%$ del total del público femenino frente a un $18 \%$ del masculino.

Tabla 1: Desglose de la audiencia por sexo (1992-2010)

\begin{tabular}{|l|c|c|c|c|}
\hline \multirow{2}{*}{\multicolumn{1}{|c|}{ Programa }} & Composición de la audiencia & \multicolumn{2}{c|}{ Cuota de pantalla } \\
\cline { 2 - 5 } & $\%$ Hombres & $\%$ Mujeres & \% Hombres & $\%$ Mujeres \\
\hline Mujeres y Hombres y viceversa & $34 \%$ & $66 \%$ & $13 \%$ & $19 \%$ \\
\hline Lo que necesitas es amor & $37 \%$ & $63 \%$ & $22 \%$ & $33 \%$ \\
\hline Su media naranja & $37 \%$ & $63 \%$ & $20 \%$ & $27 \%$ \\
\hline Xti & $37 \%$ & $63 \%$ & $13 \%$ & $15 \%$ \\
\hline I love Escassi & $39 \%$ & $61 \%$ & $10 \%$ & $14 \%$ \\
\hline Perdóname & $40 \%$ & $60 \%$ & $19 \%$ & $25 \%$ \\
\hline Vivan los novios & $41 \%$ & $59 \%$ & $20 \%$ & $26 \%$ \\
\hline Confianza Ciega & $43 \%$ & $57 \%$ & $21 \%$ & $23 \%$ \\
\hline Granjero busca esposa & $51 \%$ & $49 \%$ & $5 \%$ & $5 \%$ \\
\hline Elígeme & $51 \%$ & $49 \%$ & $5 \%$ & $4 \%$ \\
\hline Promedio total & $37 \%$ & $63 \%$ & $18 \%$ & $24 \%$ \\
\hline
\end{tabular}

Fuente: Kantar.

En términos de edad y de nivel socioeconómico (Tabla 2), los dating shows tienden a ser más populares entre la población adulta y las clases de nivel económico medio y bajo.

Tabla 2: Audiencia por tramos de edad y nivel socioeconómico (1992-2010)

\begin{tabular}{|c|c|c|c|}
\hline \multirow{2}{*}{ Criterio } & Grupo & $\begin{array}{c}\text { Composición de la } \\
\text { audiencia }\end{array}$ & Cuota de pantalla \\
\hline \multirow{4}{*}{$\begin{array}{c}\text { Por tramos de } \\
\text { edad }\end{array}$} & $4-12$ & $6 \%$ & $16 \%$ \\
\cline { 2 - 4 } & $13-24$ & $12 \%$ & $21 \%$ \\
\cline { 2 - 4 } & $25-44$ & $23 \%$ & $19 \%$ \\
\cline { 2 - 4 } & $45-64$ & $30 \%$ & $22 \%$ \\
\hline \multirow{3}{*}{$\begin{array}{c}\text { Por nivel so- } \\
\text { cioeconómico }\end{array}$} & $65+$ & $29 \%$ & $25 \%$ \\
\cline { 2 - 4 } & Bajo, medio-bajo & $\mathbf{1 0 0 \%}$ & $\mathbf{2 1 \%}$ \\
\cline { 2 - 4 } & Medio & $37 \%$ & $24 \%$ \\
\hline & Tlto, medio-alto & $12 \%$ & $20 \%$ \\
\hline
\end{tabular}

Fuente: Kantar.

Los primeros acercamientos al dating show en España consistieron en hacer preguntas o poner pruebas a parejas para descubrir la complicidad y comunicación que existía entre ellas: son los dating games o royal couple shows. Ding Dong (TVE, RTVE, 1980), Su media naranja o Luna de miel (Forta, Gestmusic Endemol, 
1992-1994) se inspiraban en un formato tímido y poco invasivo en cuanto a los efectos en la vida real de las personas: The newlywed game (ABC, Chuck Barris Productions, 1966-1974). Su media naranja preguntaba a las parejas sobre su vida de forma naíf y divertida, Ding Dong buscaba más una exposición masiva de la intimidad, incluso con secretos inconfesables, y Luna de miel introducía "acción y aventura" (Hidalgo-Marí, 2018: 630).

Después de estos primeros programas, los formatos empiezan a ser más ambiciosos y dan un salto cualitativo imitando a The dating game (ABC, 1965), producido por Chuck Barris, donde un soltero o soltera iba al programa a encontrar una pareja entre tres candidatos. Vivan los novios (Telecinco, 1991-1994), Amor a primera vista (TVE3, Gestmusic, 1991-1993) o Lo que necesitas es amor (Antena 3, Videomedia, 19931999) ya plantean ante la audiencia un cambio en la vida real de las personas: alguien busca una pareja. Sobre esa decisión empieza a construirse la relación realidad-ficción y la esencia del dating show. A diferencia de la ficción (comedia romántica o drama romántico), el reality nunca tiene un control total sobre el relato y tiene que dejar espacio a la espontaneidad de los concursantes que tienen que reaccionar con libertad en una estructura prefijada: afrontar un dilema-tomar una decisión-provocar un cambio en su vida. Cada programa organiza ese espacio de libertad impulsando la visualización de los máximos elementos posibles del cuento de hadas (historia de amor que termina bien), pero protegiendo el efecto realidad que se pacta tácitamente con la audiencia: las personas deben actuar con libertad, es decir, sin guion.

Lo que necesitas es amor fue el dating más visto de los años 90 (Tabla 3). El programa buscaba entrar en la intimidad de una relación y asistir a un momento que podía cambiar la vida de una persona. Si alguien deseaba estar o reconciliarse con alguien podía pedir ayuda al programa. El presentador del programa (Isabel Gemio, 1993-1994 o Jesús Puente; 1995-1999) hacía una visita a una persona y le entregaba un mensaje de amor en un entorno controlado: una caravana. Y le daba la opción de ir al plató o no. Algunos aceptaban y otros rechazaban esa posibilidad. Entre medias, los personajes contaban cómo se habían conocido, qué paso entre ellos o qué sentían el uno por el otro. La historia se iba construyendo hasta que terminaba en el plató con el comienzo de la relación amorosa... o su final precipitado. La posibilidad de una negativa daba más valor a la escenificación amorosa en el formato; lo hacía auténtico.

Tabla 3: Audiencias medias de los dating shows para el período 1992-2010

\begin{tabular}{|l|l|l|c|}
\hline \multicolumn{1}{|c|}{ Programa } & \multicolumn{1}{|c|}{ Cadena } & \multicolumn{1}{c|}{ Período } & Cuota de pantalla \\
\hline Lo que necesitas es amor & Antena3 & $1993-1999$ & $28 \%$ \\
\hline Su media naranja & Telecinco & $1992-1996$ & $24 \%$ \\
\hline Lo que necesitas es amor & Telecinco & $1992-1994$ & $23 \%$ \\
\hline Perdóname & TVE1 & 1994 & $22 \%$ \\
\hline Confianza ciega & Antena3 & 2002 & $22 \%$ \\
\hline Xti & Antena3 & 2003 & $14 \%$ \\
\hline Granjero busca esposa & Cuatro & 2008 & $5 \%$ \\
\hline Mujeres y Hombres y viceversa & Telecinco & $2008-2010$ & $16 \%$ \\
\hline Elígeme & Cuatro & 2009 & $4 \%$ \\
\hline I love Escassi & Telecinco & 2010 & $12 \%$ \\
\hline Total, dating shows & & $\mathbf{1 9 9 2 - 2 0 1 0}$ & $\mathbf{2 1 \%}$ \\
\hline
\end{tabular}

Fuente: Kantar

Sin embargo, los dating shows en los 90 aún no sabían explotar el potencial dramático del subgénero. El tiempo y las dificultades daban valor a un final que si se exponía fuera de contexto perdía interés humano y, por lo tanto, televisivo. Sin el proceso, sin el desarrollo dramático (dudas, reflexión, conflicto) la aproximación al cuento de hadas era más pobre. En Lo que necesitas es amor había una pequeña sección que ponía en contacto a parejas. Una persona enviaba un vídeo de presentación y el programa seleccionaba 6 posibles candidatos/ as. Luego, dejaba al autor del vídeo con sus pretendientes a solas en una cafetería de atrezo para que pudiera hablar con todos. El programa continuaba y no se mostraba nada de los posibles encuentros amorosos. Cuando el que buscaba el amor había encontrado a su pareja apretaba un botón y se escuchaba una breve sintonía, el presentador se acercaba y la audiencia descubría a quién se había elegido. Ese momento feliz quedaba impostado y desvaído; poco realista. La historia de amor quedaba reducida al final y no parecía en absoluto una historia de amor.

Otro problema de estos primeros dating shows consistía en la escasa asimilación de la mecánica de los formatos por parte de las personas que aparecían en pantalla: no sabían cuál era su papel, no sabían actuar. En Lo que necesitas es amor, una persona desconocida, llamada Isabel, queda con Isabel Gemio para ir las dos adonde está su posible pareja y decirle que le quiere. Isabel le confiesa su amor al joven y los dos se besan 
delante de las cámaras, pero todo es muy rápido, muy burocrático. Muy a pesar de Isabel Gemio, que buscaba que hubiera emoción, arrancar una película a la realidad. Al entrar ya le dice a ella: "Tienes que estar muy decidida". "Sí, sí, yo estoy muy decidida", replica la enamorada. Y luego cuando le confiesa su amor a la pareja y este se queda pasmado: "Ayúdale un poquito" o "Dale un beso o algo para que no se quede así". La dirección de la presentadora fue estéril, porque las personas se guardaron las palabras y las emociones. Aún no eran personajes; les intimidaban las cámaras y les costaba entender su rol y localizar qué era lo auténtico para la televisión, para el formato.

En Perdóname (TVE, John de Mol y Joris van Ooijen, 1994), María Teresa Campos se encontraba con el mismo problema. En el plató una mujer había pedido perdón por sus infidelidades a su marido, y él la había perdonado: todo estaba contado ya en un reportaje previo. No había espontaneidad, ni frescura. Por eso Campos intentaba empujar los límites del formato: "Pero me gustaría saber: si usted hubiera engañado en estos 17 años a su mujer, si usted sería capaz de hacer lo que está haciendo ella. Y, en cualquier caso, [...] ¿usted no ha engañado nunca a su mujer?" El marido estaba confuso. Eso se salía del guion pactado. Al final sólo pudo balbucear un "nunca". Y con la ayuda de su mujer, que le decía: "no pasa nada". Y el tema quedaba bloqueado. $\mathrm{Ni}$ el reality ni sus protagonistas entendían aún el valor de la espontaneidad emocional.

Estos primeros programas sobre el amor mostraban poca emoción, intensidad y orden, pero no toda la culpa era del formato. Para hacer surgir el conflicto y las emociones de forma espontánea, y que no pareciera mala ficción, tenía que haber cambios en la mecánica, pero también en los participantes: los enamorados aún no entendían las reglas del amor televisivo. Tenían demasiadas líneas rojas en la expresión de su intimidad, demasiado pudor. Paradójicamente, años después, un bloguero criticaba a los concursantes de un dating show justo por lo contrario; por una compresión excesiva de esas reglas y de tener plena consciencia de estar actuando para un programa, lo cual restaba verdad: "Entre gente normal, todo debería haber quedado en un roce sin más, pero los personajes que van a estos programas... ya se sabe” (Anon., 2009).

\section{Gran Hermano y su influencia en el dating show}

GH cambió la dimensión y la confianza en los realities. Su éxito hizo que muchos de sus elementos aparecieran en otros formatos buscando la misma conexión con la audiencia. El concurso de Endemol también significó un impulso y un descubrimiento para la evolución de los dating shows: después de la primera cita, la historia continuaba. Cuando apareció GH la posibilidad de ofrecer encuentros sexuales en un programa que se jactaba en mostrar la vida sin filtros y en estado puro concentró atención y críticas: “aquí huele a sexo [...] estos chicos de Gran Hermano garantizan la tensión sexual... aunque no parece que vayan a poder aguantar demasiado tiempo sin resolverla" (Amela, 2000: 11). Se acusaba al programa no sólo de favorecer o esperar el sexo entre concursantes, sino de necesitarlo para que el formato tuviera interés: “[...] los guionistas no lo tendrán fácil para mantener interesada a la audiencia si no hay "tetas, más tetas" (Baget, 2000: 10). Pero el contenido sexual no apareció... al menos en GH1, curiosamente la edición más vista. Las cámaras estaban preparadas, pero los amantes aún no.

Sin embargo, eso no significaba que al formato no le favoreciera el sexo entre concursantes, aunque no con el propósito que intuían sus detractores. El valor del sexo no estaba en el acto en sí, sino en su capacidad para influir en el desarrollo narrativo: las posibilidades dramáticas se multiplicaban y el relato era mejor. En GH9 la trama amorosa entre Melania y Piero ganó en profundidad y cambios gracias a una hora en privado sin cámaras. La pareja pasó más tiempo junta que antes, lo cual despertaba el recelo de media casa. Amor (nombre de una concursante) le da a Melania su visto bueno a su relación con Piero cuando sabe que ya hubo sexo entre ellos. Melania deja a Piero y luego vuelve lamentándose de no ser "lo bastante fuerte para rechazarlo". Amor hace campaña contra Piero para que lo expulsen (antagonista), mientras Melania lamenta no dedicarle tanto tiempo a su amiga por estar con él (conflicto interior). Y cuando la audiencia echa a Piero, Melania llora en la casa por su pérdida (Santana, 2011: 296-299). Sin la hora sin cámaras, los concursantes no hubieran profundizado tanto en su relación, ni tampoco habría llegado el conflicto interior, ni la intensidad de las reacciones hubieran sido iguales. El programa no tenía el control total de la historia, pero creaba las condiciones y tenía el tiempo para que sucediera. Algo similar sucedió con el anti-dating show: Confianza ciega (Antena 3, Zeppelin TV, 2002). El programa encerraba a parejas y ponía a prueba su fidelidad, haciendo que convivieran por separado con mujeres y hombres atractivos que tenían como único propósito seducir a los concursantes. Se buscaba la historia de desamor a través de la distancia, la relación con otras personas y las dudas que provocaban los vídeos que veían sobre sus parejas, y que no sabían si estaban manipulados o no (Anon., 2017).

Los dating shows asimilaron rápidamente todos estos conceptos narrativos (Puebla-Martínez, Magro-Vela y Fernández-Valera, 2018) y buscaron su propia fórmula para poseer una historia de amor más compleja. El dating show necesitaba crear tiempo: hacer un relato en torno a una decisión. Casi quince años después de Lo que necesitas es amor, MyHyV explotaría de forma diferente la declaración de amor usando citas e introduciendo como elemento estructural las opiniones sobre la relación. El programa, basado en el formato italiano Uomini e donne (Canale 5, Fascino PGT, 1996-), se construía en torno a la necesidad de hacer que el 
objetivo dramático de los protagonistas fuera difícil de lograr, como en una película (Sanz-Magallón, 2007). En MyHyV había un hombre o una mujer (tronista), que tenía citas con diferentes candidatos que estaban en el plató y que se postulaban como pareja, todos ellos guapos o guapas y de cuerpo perfecto (pretendientes). El/La tronista decidía si se quedaban como pretendientes o directamente eran rechazadas/os. Las citas eran grabadas y luego se analizaban en el plató, se preguntaba cómo se sentían y se juzgaba lo que habían hecho. Un/a tronista podía durar 3-4 meses y el criterio para no admitir más pretendientes lo decidía el share de audiencia: sólo se cerraba el casting cuando el/la tronista no daba más juego, es decir, si no generaba interés dramático, si la película ya no interesaba. En todo este proceso de citas sin fin y evolución de las relaciones en paralelo estaba el placer de juzgar. Opinaba el público, los "consejeros", que eran personas de confianza que orientaban a los/las tronistas, y, por supuesto, opinaba la presentadora, que abandonaba toda neutralidad para agitar, preguntar, orientar la discusión, presionar y emitir juicios sobre lo que hacían los jóvenes.

Elígeme, basado en el formato australiano Take me out (Seven Network, Fremantle Media, 2008-2009), tenía una estructura similar, pero con matices. Lo presentaba Carlos Baute y consistía en un soltero que iba al plató a buscar una cita con 24 posibles mujeres que se situaban detrás de un atril en un semicírculo. Si el atril se iluminaba o se apagaba significaba que la mujer estaba interesada en la cita o definitivamente desistía. Primero, el soltero se presentaba diciendo su nombre y edad. Sólo por su físico y apariencia, las mujeres activaban por primera vez el atril. Luego, la operación se repetía dos veces más después de que el soltero diera más datos o mostrara alguna habilidad. Así, las mujeres decidían en tres ocasiones si les gustaba el hombre o no. De entre las que quedaban con la luz del atril encendido, el soltero escogía a tres, y ahora él les hacía preguntas y finalmente optaba por una de ellas y tenía una cita, que se grababa.

Las mujeres podían permanecer en Elígeme durante un máximo de dos semanas optando por completar todas las fases del programa (Rizzo, 2009). Cada día aparecían tres solteros y se repetía este proceso. En comparación con MyHyV, Elígeme apenas explotaba las citas y los comentarios. Baute intentaba ser espontáneo, descarado y provocador, pero siempre con los límites claros. Él decidía con quién hablaba y cuándo y marcaba el tono, lo cual hacía todo más monótono, menos sorprendente. Pero la gran diferencia con MyHyV era el tiempo en el plató y las citas. En Elígeme, las citas no permitían desarrollar la relación, simplemente la culminaban. Y no eran estructurales en el programa, no lo definían:

Después se supone que tienen una cita de la cual sólo se ven 5 segundos, y se ve que hay un encuentro al que se presentan si quieren algo con esa persona, y son otros 3 segundos únicamente [...]. A mí este programa me parece una tontería donde se podrían ahorrar los vídeos y las demostraciones de habilidades, porque lo único que se mira es el físico, tanto hacia un lado como hacia otro, y total, para luego no ver nada de la cita, no tiene encanto ninguno (Teysis, 2009).

El dinamismo y el interés en MyHyV está en las citas: el momento sin guion y, por tanto, el momento donde hay más libertad por parte de las personas, más autenticidad. Lo mismo sucede con First dates (Cuatro, Eyeworks-Cuatro Cabezas, 2016-), basado en el formato británico del mismo nombre, que edita hábilmente las citas de personas afines para construir sus desavenencias o complicidades. El tiempo de cita, lo que dura una comida entre ambos, desemboca en un momento que puede afectar a la vida real: hay que decir, delante de la otra persona, si se acepta continuar la relación y luego esperar la respuesta de su compañero/a de cita por si coincide. El formato también juega con diferentes verdades: graba la opinión de los posibles enamorados sin la otra persona delante. Los matices pueden ser grandes, incluso pueden mentir: en este caso la mentira también es un momento de verdad plena, porque elegir palabras amables para no querer hace daño o simplemente esconderse resulta tan real en las relaciones como las clásicas declaraciones amorosas. En el tiempo de cita tampoco hay guion; está lleno de verdad. El espacio de la ficción es absolutamente decisivo al obligar a esa confesión de emociones, pero esas emociones no están en un guion, sino que están por escribir y dependen de la experiencia vivida por las dos personas.

En MyHyV, la cita tiene un valor diferente porque en su estructura no es finalista: después de una cita puede haber más y también puede haber citas con otras personas. Se multiplican los puntos de vista, lo cual conduce de forma directa al conflicto. Todas las citas se ven y se juzgan. Muchas personas hablan de ellas, no sólo los protagonistas. Eso plantea dudas, reflexiones, arrepentimientos, reafirmaciones y fuego cruzado entre personas que se enfrentan por logar un objetivo que no pueden compartir: más conflicto. Las historias se crean, se desarrollan (con más citas) y buscan, lentamente, un final con todos los componentes dramáticos necesarios. En MyHyV todo gira en torno a la cita y a la posibilidad de poder juzgar la autenticidad del amor a través de lo que hacen unos y otros: el reality juzga el reality. En un tono menos agresivo, ¿Quién quiere casarse con mi hijo? (Cuatro, Cuatro Cabezas, 2012), ¿Quién quiere casarse con mi madre? (Cuatro, Cuatro Cabezas, 2013) o Un príncipe para Corina (Cuatro, Cuatro Cabezas, 2013) usaron la serialidad para conquistar ese tiempo que permite a la audiencia ver el inicio de una relación por dentro: dudas, conflictos, miedos, presiones o consejos. 


\section{La autenticidad y el reality}

Otros formatos buscaron controlar las citas desde otro enfoque, sumando el encierro de GH con características del formato americano The Bachelor (ABC, Next Entertainment-Telepictures Productions, 2002-). Xti (Antena3, Zeppelin TV, 2003) y I love Escassi (Telecinco, Pulso, 2010) tenían estructuras similares: uno o varios solteros deseables y muchas mujeres queriendo ser su pareja y encerradas en un mismo lugar para potenciar la competitividad entre ellas. En ambos dating shows, la distribución del espacio era muy reveladora. En Xti, los tres hombres disponían de habitación propia con jacuzzi, cama de matrimonio con colcha que aparentaba piel de leopardo, internet, terraza... Mientras las doce mujeres se tenían que hacinar en dos habitaciones con seis camas cada una y dos baños a compartir. El reality tenía un tono fácil de reconocer: "Antena 3 monta un harén para 3 solteros en Xti" (Gómez, 2003). Un grupo de psicólogos y "profesionales especializados" hizo el casting de las doce mujeres: "realizamos entrevistas en profundidad porque queríamos gente que realmente deseara encontrar pareja, y su motivación de concursar no fuera la fama o el dinero" (Anon., 2003). Se buscaba autenticidad, pero la audiencia no respondió y el programa se canceló.

I love Escassi ya estaba marcado por la personalidad de seductor irredento del jinete Álvaro Muñoz Escassi. A priori no era fácil creer que Escassi buscara pareja: la falta de autenticidad estaba ya en el origen. Sin haberse estrenado aún el programa, la crítica intuía las debilidades y las fortalezas del formato: "se trata de un programa 'timo', porque ya está grabado y ya se sabe que Escassi no conseguirá poner 'remedio' a su soltería. [...] Aun así, esta película ¿porno? tiene posibilidades de salir bien parada en la taquilla del 'share"' (Anon., 2010a). El primer programa obtuvo un share de audiencia bastante pobre (9\%) y la cadena intentó reaccionar incluyendo un toque de MyHyV y del formato docu-show. Jesús Vázquez comentaba lo que sucedía en voz en off y se organizaba un debate final con invitados (Anon., 2010b). La audiencia no remontó a pesar de que Escassi se acostó con tres de las concursantes y sumó un largo corolario de momentos eróticos. Las quejas a la falta de autenticidad fueron habituales: desde la crítica -"El jinete sevillano no es un tipo genuino" (Álvaro, 2010) - hasta la ganadora del concurso, que admitió que Escassi le dijo que no estaba enamorado de ella "aunque podría estarlo" (Anon., 2013).

Las audiencias de un dating show no se ven afectadas porque aparezcan lugares o acciones poco verosímiles comparadas con una cita normal (Ferris et al, 2007), pero sí hay distanciamiento en los espectadores si no encuentran verosimilitud emocional o si descubren que el amor se crea ad hoc, ya sea por la voluntad de las personas por ser un buen personaje o por presiones de producción para hacer un buen producto (Klassen, 2014; Lowry, 2018). La realidad es parte del pacto con la audiencia en un reality y exige su espacio. Granjero busca esposa era un formato más inspirado en The Bachelor, pero tenía algo diferente: "le sobraba algo que los otros [dating shows] no llegaban ni a oler: verdad" (Piñeiro, 2015). Granjero busca esposa, adaptación del formato británico The farmer wants a wife (ITV, Thames Television, 2001), desarrollado después en Australia por Fremantle Media, partía de un punto de mayor autenticidad. Las personas que buscaban pareja eran ganaderos y vivían en el campo, donde la oportunidad de relaciones era escasa y los trabajos, muy absorbentes: eran solitarios por circunstancias. Eso arrastraba una sensación de verdad que a priori no tenían ni los prototipos de modelos de MyHyV, ni los personajes del corazón. La impresión de autenticidad afectaba al reality sin que el formato en sí mismo fuera demasiado diferente a otros. Las diferencias en lo emocional eran pequeñas comparadas con otros dating shows. Estaban las dudas, los juegos, los desencuentros, las comparaciones, la opinión de los otros (familiares), los celos, el posible enamoramiento... Los primeros concursantes temían la puesta en escena del dating show -la parte de ficción-e intentaban, como tantos concursantes, defenderse de ella: "Yo no quería que fuera un Gran Hermano o algo parecido. Me dijeron que era respetuoso con la gente y me convencí", confesaba Nides Riesco. "Yo voy en serio, quiero encontrar una compañera", decía José Antonio González (Gómez, 2008). Independientemente de su sinceridad, la proyección de verdad sobre el reality, sólo por la elección de los protagonistas, era mayor. El formato dejó de emitirse en 2011 y cuando se volvió a programar en 2016, los primeros "granjeros" se quejaban de la pérdida de autenticidad de la quinta edición: "[...] ha degenerado mucho y ha perdido la naturalidad; [...] ahora los granjeros se lían con todas y no hay respeto, se parece más a Gran Hermano o Mujeres, Hombres y Viceversa" (Pedro Gonzáles, $3^{\mathrm{a}}$ edición) o "ahora es más un programa de espectáculo y de entretenimiento" (Nides Riesco, 1ª edición) (Anon., 2016).

\section{Conclusiones}

Los dating shows son un subgénero dentro de los realities que ofrecen a la audiencia historias de amor lo más realistas posibles, aunque sin olvidar que, igual que sucede en las películas, el realismo existe "como apoyo al significado, no como objeto en sí mismo" (Bordwell, Staiger y Thompson, 1997: 21): la expresión de una representación veraz no es absoluta, pero eso no significa que sea percibida como falsa. El subgénero pasó de preguntar curiosidades sobre las relaciones amorosas de las personas o comprobar su grado de complicidad a estar allí cuando comenzaba la historia de amor. Y, después, a intentar poseer esa historia... con ayuda de la ficción. La parte de ficción en un dating show significa imponer una sucesión de momentos (orden o 
estructura) en los cuales las personas expresan emociones o tienen la oportunidad de vivir experiencias que pueden afectar a su vida real. Esta parte de ficción está conscientemente limitada. Cada situación que genera un dating show impone un margen de reacciones posibles, pero las personas tienen que decidir según su libertad y sus emociones.

La ficción en una serie o en una película tiene un control absoluto de los personajes; la ficción en el reality condiciona el comportamiento de las personas, pero no decide sobre ellas, no puede invadir espacios de libertad que corresponden al individuo. Los formatos defienden la invisibilidad de la ficción y dejan espacio para que suceda lo real, lo no previsto por guion, lo espontáneo. Esa espontaneidad tampoco significa que el concursante puede hacer lo que desee. En los primeros formatos de los años 90, las personas elegían no hablar, se bloqueaban o disimulaban para no mostrar sus emociones. En la evolución del género fue muy importante el aprendizaje televisivo. Las personas que acudían a los dating shows empezaron a entender y a comprometerse con cada uno de los formatos, lo cual reducía su margen de libertad y limitaba sus acciones al marco de verosimilitud definido por la dinámica y las situaciones de cada programa. Nacía el "personaje": la persona consciente de la mirada de la audiencia y de su propio rol dentro de la estructura del programa.

La evolución del dating show demuestra que la expresión de la realidad no es pura en un reality y tiene límites marcados por la necesidad de crear un producto entretenido para la televisión; esta necesidad incluso genera excesos, tentaciones de recuperar el viejo control total de la ficción. Esta deriva conecta con las argumentaciones de varios autores sobre la quiebra o la dudosa representación veraz que hacen estos programas y su tendencia a la performance, es decir, a la actuación no natural de las personas que se saben dentro de un reality (Imbert, 2008a; León, 2013; Puebla-Martínez, Magro-Vela y Fernández-Valera, 2018). Sin embargo, las audiencias responden positivamente a la autenticidad en los realities (Jones, 2003; Papacharissi; Mendelson, 2007), es decir, a sentir que las personas actúan movidas por sus sentimientos o sus deseos. Los formatos de dating show demuestran ser progresivamente más invasivos, mostrando más momentos de las relaciones íntimas entre las personas, lo cual obliga a nuevas estructuras: nueva ficción. Aun así, la audiencia también busca y encuentra una representación veraz en ellos. La conexión con lo real está en las acciones o decisiones que toman los concursantes "de forma libre e inesperada" (Bignell, 2005: 115): ese es el alma del reality. A pesar de que la realidad está condicionada en los dating shows, igual que está la ficción, los formatos siguen preservando lo más importante: la verosimilitud emocional a través de acciones libres ante determinadas circunstancias o personas (Kavka, 2008). El desarrollo histórico de los dating shows demuestra que hay una mayor puesta en escena: más momentos de las relaciones retocados u organizados por la ficción. Sin embargo, ese aumento de la performance no daña la autenticidad en la medida en que son evidentes importantes dosis de libertad en las personas que derivan en efectos para su vida real.

\section{Referencias bibliográficas}

Álvaro (2010). "I love Escassi”. La página definitiva, 21-05-2010. Disponible en: http://www.lapaginadefinitiva. com/2010/05/21/i-love-escassi/

Amela, V. (2000). "La primera noche de Gran Hermano". La Vanguardia, 25-04-2000. Disponible en : http://hemeroteca. lavanguardia.com/preview/2000/04/25/pagina-11/34056911/pdf.html?search=la $\% 20$ primera $\% 20$ noche $\% 20 \mathrm{de} \% 20$ gran $\% 20$ hermano

Anon. (2003). "Paula Vázquez presentará el reality show de solteros 'Xti’ a partir del 2 de marzo". Verteletv! 19-2-2003. Disponible en: http://vertele.eldiario.es/verteletv/actualidad/Paula-Vazquez-presentara-reality-solteros_0_343465656. html

Anon. (2009). "Menos mal que se gustaban". El blog del becario. 20minutos, 9-8-2009. Disponible en: https:// blogs.20minutos.es/becario/2009/08/09/menos-mal-se-gustaban/comment-page-2/

Anon. (2010a). "Así liga Álvaro Muñoz Escassi”. El Confidencial, 26-2-2010. Disponible en: https://www.vanitatis. elconfidencial.com/multimedia/video/television/2010-02-26/asi-liga-alvaro-munoz-escassi 690403/

Anon. (2010b). "La solución 'a la desesperada' de Telecinco para reflotar I love Escassi incluye falsos directos en 'Sálvame' con la intervención del jinete”. El Confidencial Digital, 5-3-2010. Disponible en: https://www.elconfidencialdigital. com/articulo/medios/desesperada-Telecinco-Escassi-Salvame-intervencion/20100305000000057940.html

Anon. (2013). "Cristina Veiga: "Mientras estuve con Escassi él no dejó de tener relaciones con otras". Telecinco. Guía TV, 11-6-2013. Disponible en: https://www.telecinco.es/salvame/2013/junio/11-06-2013/Cristina-Veiga-Escassirelaciones-mujeres_0_1618575455.html

Anon. (2016). "Granjero busca esposa: ¿qué fue de Pedro y Nides? La Opinión de Zamora, 12-11-2016. Disponible en: https://www.laopiniondezamora.es/comarcas/2016/11/11/granjero-busca-esposa-pedro-nides/964804.html

Anon. (2017). "Confianza ciega, el polémico programa que marcó las relaciones de pareja”. La Vanguardia, 9-12-2017. Disponible en: https://www.lavanguardia.com/television/20171209/433471168211/confianza-ciega-reality-parejasantena-3-nube.html

Baget, J.M. (2000). “Los chicos montan su ONG particular”. La Vanguardia, 27-4-2000. Disponible en: http://hemeroteca. lavanguardia.com/preview/2000/04/27/pagina-10/34061649/pdf.html?search=los\%20chicos\%20montan\%20su\%20 ONG\%20particular 
Bignell, J. (2005). Big brother. Reality tv in the twenty-first century. Nueva York-Londres: Palgrave McMillan. ISBN: 978 0230508361

Bordwell, D.; Staiger, J.; Thompson, K. (1997). El cine clásico de Hollywood: estilo cinematográfico y modo de producción hasta 1960. Barcelona: Paidós. ISBN: 8449301297

Corner, J. (2009). "Performing the real: documentary diversions (with afterword)". En: Murray, Susan; Ouellette, Laurie (Eds), Reality TV. Remaking Television Culture. Nueva York - Londres: New York University Press, pp. 44-64. ISBN: 9780814757345

Cummings, D. (Ed.) (2002). Reality TV: How real is real? Oxford: Hodder and Stoughton. ISBN: 9780340857359

Ellis, J. (2009). "The performance on television of sincerely felt emotions". The annals of the American academy of political and social science, v. 625, n. 1, pp. 103-115. https://doi.org/10.1177/0002716209339267

Ferris, A.L.; Smith, S.; Greenberg, B.; Smith, S. (2007). "The content of reality dating shows and viewer perceptions of dating". Journal of Communication, v. 57, pp. 490-510. https://doi.org/10.1111/j.1460-2466.2007.00354.x

Gómez, I. (2008) “Granjero soltero busca...". La Opinión de Zamora, 20-9-2008. Disponible en: https://www. laopiniondezamora.es/comarcas/2008/09/20/granjero-soltero-busca/302879.html

Gómez-Madrid, A. (2003). “Antena 3 monta un harén para 3 solteros en Xti”. El periódico. Extremadura, 2-3-2003. Disponible en: https://www.elperiodicoextremadura.com/noticias/television/antena-3-monta-haren-tres-solteros-Xti_41948.html

Gordillo, I. (2009). Manual de narrativa televisiva. Madrid: Síntesis. ISBN: 9788497566407

Gray, J. (2009). "Cinderella burps: gender, performativity, and the dating show”. En: Murray, Susan \& Ouellette, Laurie (Eds.), Reality TV: remaking television culture. Nueva York: New York University Press, pp. 260-277. ISBN: 978 14 79830879

Hidalgo-Marí, T. (2018). "Pasado, presente y futuro del dating show en España: una panorámica del subgénero de telerrealidad". Estudios sobre el Mensaje Periodístico, v. 24, n. 1, pp. 623-641. https://doi.org/10.5209/ESMP.59970

Hill, A.; Palmer, G. (2002). "Big Brother". Television \& New Media, v. 3, n. 3, pp. 251-254. https://doi.org/ $10.1177 / 152747640200300301$

Imbert, G. (2008a). "Bienvenidos al desierto de lo hiperreal”. El País, 1-11-2008. Disponible en: https://elpais.com/ diario/2008/11/01/opinion/1225494004_850215.html

- (2008b). El transformismo televisivo. Postelevisión e imaginarios sociales. Madrid: Cátedra. Signo e imagen. ISBN: 8437624991.

Jones, J.M. (2003). "Show Your Real Face”. New Media \& Society, v. 5, n. 3, pp. 400-421. http://dx.doi.org/ $10.1177 / 14614448030053006$

Kantar (1990-2010). Empresa de Medición de audiencias. https:/www.kantarmedia.com/es/sobre-nosotros/kantar

Kavka, M. (2008). Reality television, affect and intimacy: reality matters. Nueva York: Palgrave Macmillan. ISBN: 978 0230545502.

Kilborn, R. (1994). "How real can you get? Recent developments in "reality" television". European Journal of Communication, v. 9, n. 4, pp. 421-439. http://dx.doi.org/10.1177/0267323194009004003

Klassen, A. (2014). "I was on reality tv: behind the scenes secrets of faking real life". Bustle, 18-2-2014. Disponible en: https://www.bustle.com/articles/15280-i-was-on-reality-tv-behind-the-scenes-secrets-of-faking-real-life

León, B. (2009). "Introducción”. En: León, B. (Coord). Telerrealidad. El mundo tras el cristal. Sevilla-Zamora: Comunicación Social Ediciones y publicaciones. ISBN: 9788492860081

- (2013). "Realidad y ficción en la 'telerrealidad"”. En: Gutiérrez, R. (Coord.). Poéticas de la persona. Creación, responsabilidad y vigencia en la Comunicación Pública y la Cultura. Salamanca: Comunicación social, pp. $229-307$. ISBN: 9788415544623

Lowry, B. (2018). "Bachelor bargain: reality tv fans choose to suspend disbelief". CNN. Entertainment, 15-3-2018. Disponible en: https:/edition.cnn.com/2018/03/15/entertainment/reality-tv-bachelor/index.html

Papacharissi, Z.; Mendelson, A. (2007). "An exploratory study of reality appeal: uses and gratifications of reality tv shows". Journal of Broadcasting \& Electronic Media, v. 51, n. 2, pp. 355-370. http://dx.doi.org/10.1080/08838150701307152

Piñeiro, R. (2015). “Todos los 'realities' emitidos en España, ordenados de peor a mejor”. Vanity Fair, 16-7-2015. Disponible en: https:/www.revistavanityfair.es/cultura/entretenimiento/articulos/reality-shows-espana-rankingmejor-a-peor-gran-hermano-supervivientes-alaska-y-mario-gandia-shore/21135

Puebla-Martínez, B.; Magro-Vela, S.; Fernández-Valera, J. (2018). "Funcionalidad de los componentes narrativos cinematográficos en los nuevos formatos televisivos: los dating shows". Anàlisi: Quaderns de comunicació $i$ Cultura, v. 59, pp. 105-119. https://doi.org/10.5565/rev/analisi.3113

Rizzo, C. (2009), "Soltero busca ligue”. 20minutos, 30-3-2009. https://www.20minutos.es/noticia/460017/0/carlos/baute/ eligeme/

Santana, G. (2011). Gran Hermano. Diario de una guionista. Madrid: Anaya. ISBN: 8441530238

Sanz-Magallón, A. (2007). Cuéntalo bien. El sentido común aplicado a las historias. Madrid: Plot. ISBN: 9788486702 786.

Teysis (2009). "Elígeme”. ...Y después de todo..., 14-4-2009. Disponible en: https://teysis.wordpress.com/2009/04/14/ eligeme/ 